Revue des patrimoines

\title{
Effets paysagers des transformations agricoles et rurales dans le sud du pays d'Auge
}

\section{Philippe Madeline}

\section{(2) OpenEdition}

1 Journals

\section{Édition électronique}

URL : http://journals.openedition.org/insitu/2565

DOI : 10.4000/insitu.2565

ISSN : 1630-7305

\section{Éditeur}

Ministère de la Culture

Référence électronique

Philippe Madeline, « Effets paysagers des transformations agricoles et rurales dans le sud du pays d'Auge », In Situ [En ligne], 7 | 2006, mis en ligne le 18 avril 2012, consulté le 14 novembre 2019. URL http://journals.openedition.org/insitu/2565; DOI : 10.4000/insitu.2565

Ce document a été généré automatiquement le 14 novembre 2019.

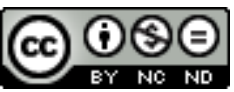

In Situ Revues des patrimoines est mis à disposition selon les termes de la licence Creative Commons Attribution - Pas d'Utilisation Commerciale - Pas de Modification 4.0 International. 


\title{
Effets paysagers des transformations agricoles et rurales dans le sud du pays d'Auge
}

\author{
Philippe Madeline
}

1 Dans le cadre d'une enquête interdisciplinaire intitulée « Autour de Camembert de l'an Mil à nos jours » menée à l'initiative du « Pôle Rural » de la Maison de la Recherche en Sciences Humaines de l'Université de Caen-Basse-Normandie ${ }^{1}$, l'analyse des transformations agricoles et rurales sur la longue durée mobilise une équipe de chercheurs en sciences sociales: archéologie, géographie, histoire, sociologie, linguistique. Dans cet espace emblématique de la Normandie, la zone méridionale du pays d'Auge, convertie au couchage en herbe à partir du XVII ${ }^{e}$ siècle, reste peu étudiée malgré la richesse des sources anciennes et les transformations de l'économie agricole des derniers siècles. Connue avant tout pour son fromage et la spécificité de son architecture, elle constitue un support médiatique privilégié à travers les clichés des constructions traditionnelles en pan de bois qui composent la cour augeronne. L'image du pays d'Auge, véritable archétype d'une campagne préservée des transformations agricoles et rurales $\mathrm{du} \mathrm{XX}^{\mathrm{e}}$ siècle, est encore largement utilisée par les médias, les professionnels $\mathrm{du}$ tourisme et l'industrie agroalimentaire. Le décalage entre cette image et l'état actuel constitue le point de départ d'une interrogation sur les transformations paysagères à l'œuvre depuis plusieurs décennies.

Pour répondre à cette question, un travail de terrain sur la commune de Camembert, a donné lieu au repérage systématique des unités bâties, à leur identification et à l'évaluation de l'état des constructions. Cet état actuel du bâti a fait l'objet d'une comparaison avec les différents supports cartographiques (cadastre de 1938 actualisé, cadastre napoléonien) et iconographiques (plan terrier de 1775). Au total, des documents de synthèse permettent d'apprécier l'évolution et les transformations des constructions dans un contexte paysager en transformation. 


\section{Quelques caractéristiques du bâti traditionnel dans le sud du pays d'Auge}

Les constructions traditionnelles du pays d'Auge sont le reflet de l'utilisation des matériaux en provenance du sol et du sous-sol : le bois pour l'ossature des murs en pan de bois, l'argile crue pour le torchis et cuite pour la brique et la tuile plate, le silex pour les moellons. C'est surtout l'association de ces matériaux, différente selon les usages et les époques, qui est la source d'une unité architecturale dont la valeur esthétique participe à la renommée du pays d'Auge. L'utilisation de nouveaux matériaux, à l'origine du renouvellement de la palette de couleurs, n'est pas un phénomène récent. Si l'on prend l'exemple des matériaux de couverture des maisons d'habitation, les toits de chaume ont été remplacés dès le XVII siècle par la tuile plate d'origine locale, ellemême remplacée par l'ardoise à la fin du XIX ${ }^{\mathrm{e}}$ siècle. Ces matériaux sont largement concurrencés depuis le milieu du $\mathrm{XX}^{\mathrm{e}}$ siècle par la tuile mécanique et l'ardoise synthétique.

Figure 1

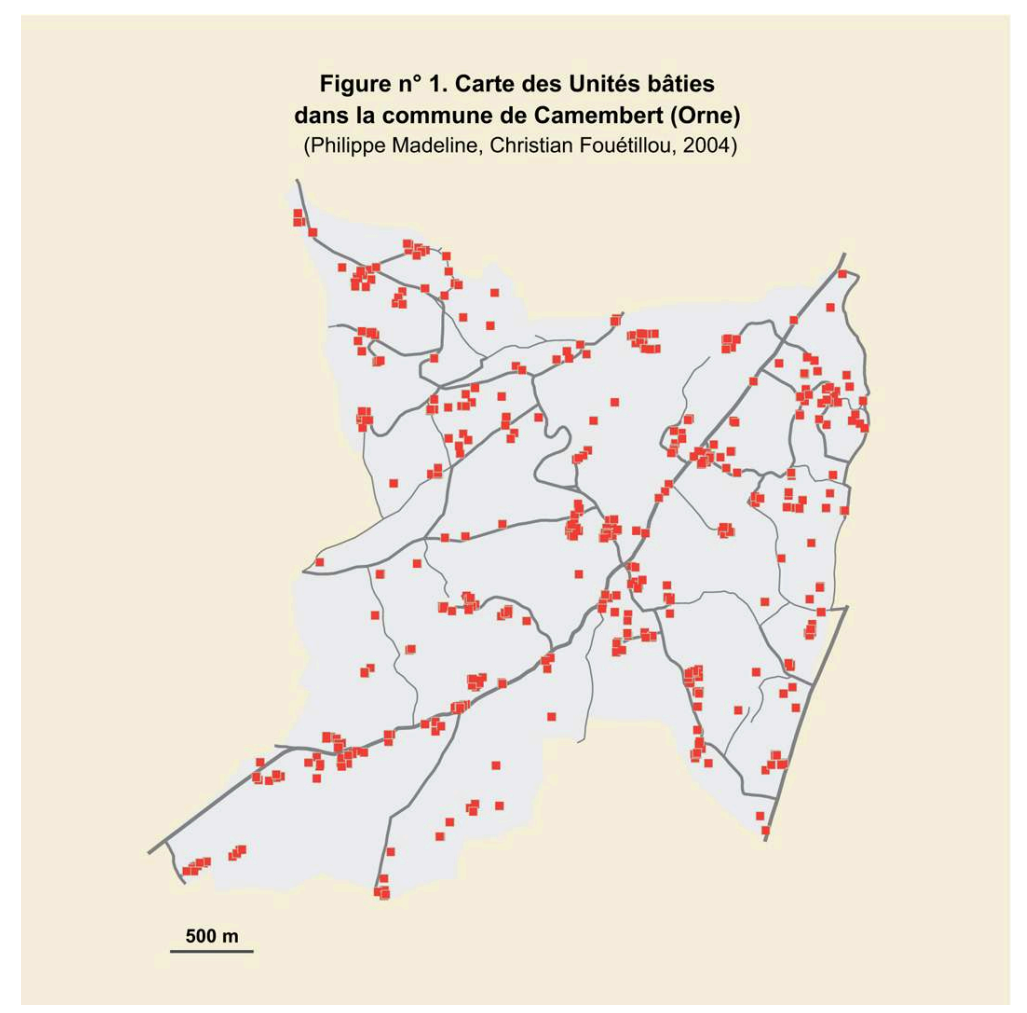

Carte des unités bâties dans la commune de Camembert (Orne)

Cartes P. Madeline - C. Fouétillou (C) P. Madeline - C. Fouétillou, 2004

La répartition de l'habitat en hameaux dispersés suit grossièrement l'orientation de la vallée de la Viette selon un axe sud-ouest/nord-est le long de la route départementale 246 et le long des petites vallées incisées par des affluents de cette rivière (fig. $\mathbf{n}^{\circ} \mathbf{1}$ ). Dans une topographie assez mouvementée, la majorité des constructions se situe à flanc de coteau sur les versants les moins exposés aux intempéries du nord-ouest. Dans un herbage complanté de pommiers hautes tiges désigné sous le terme de «clos augeron ", l'organisation des bâtiments de l'économie agricole traditionnelle s'articule 
autour de l'habitation et de différents bâtiments à la fonction bien définie : l'écurie, la grange, la charetterie, le pressoir, la boulangerie, la bouillerie, la fromagerie sans oublier, en dehors du clos, des bâtiments isolés dans les herbages. Par leur nombre, les bâtiments répondant à une fonction agricole ancienne ou récente sont deux fois plus nombreux que les maisons d'habitation: ils apparaissent alors comme des éléments marquants des paysages augerons (fig. $n^{\circ} 2$ ).

Figure 2



Paysage de la commune de Camembert. Au second plan : la ferme de Beaumoncel Phot. Inv. J.-Cl. Jacques @ Inventaire général, ADAGP, 1991

\section{L'inadaptation du bâti traditionnel aux conditions agricoles modernes}

5 Parmi les évolutions de la vie sociale et économique contemporaine, deux aspects éclairent les transformations paysagères du sud du pays d'Auge: l'évolution de la population et les transformations agricoles.

6 A l'échelle du canton de Vimoutiers, la diminution de la population sur deux siècles s'exprime d'abord à partir de la densité : 56 habitants au $\mathrm{km}^{2}$ en 1806, moins de 20 au dernier recensement. Pour la commune de Camembert, la population a été divisée par trois : un peu moins de 700 habitants au début du XVIII siècle et moins de 200 au dernier recensement. On remarque cependant, à l'image de la quasi-totalité des communes rurales depuis le milieu des années 70 , un retournement de tendance avec la fin du recul de la population: 176 habitants en 1975, 184 en 1990, 197 en 1999. Cette inversion des courbes se répercute sur le nombre de résidences principales: 62 en 1968 et 75 en 1999 alors que se multiplient les résidences secondaires : 6 en 1968 et 31 aujourd'hui. Avec l'augmentation de la population permanente, de nouvelles constructions apparaissent et les résidents secondaires participent activement au recyclage du bâti traditionnel, laissé vacant par une agriculture en mutation.

7 Au-delà des effets identifiés pour l'ensemble des régions agricoles et plus spécifiquement pour les régions de bocage, c'est-à-dire la diminution du nombre des 
exploitations (38 en 1940 et 21 en 2000), la mécanisation, la simplification parcellaire et la réduction des vergers haute tige, c'est surtout l'inadaptation des bâtiments agricoles aux conditions de l'agriculture moderne qui retient notre attention. Elle n'est pas spécifique au pays d'Auge mais du fait de la structure du bâti et des matériaux de construction utilisés, les transformations sont radicales car le réemploi dans le cadre d'une agriculture actuelle est impossible. Quelles sont les conséquences de ces transformations sur le patrimoine architectural de la commune?

\section{Un patrimoine architectural « dégradé »}

Les transformations sociales et économiques des campagnes du sud pays d'Auge ont des effets architecturaux et paysagers qui bouleversent une image bien ancrée dans l'imaginaire collectif.

Les évolutions combinées de la démographie et de l'agriculture permettent d'abord d'identifier la disparition progressive et inéluctable d'une relative unité architecturale augeronne. La concurrence des matériaux traditionnels par de nouveaux matériaux est le premier élément d'explication: en 1992, le recensement effectué par l'Inventaire général indiquait que plus de $65 \%$ des maisons et des fermes étaient constituées de pan de bois et entre 12 et $20 \%$ recouvertes de tuile plate ${ }^{2}$. L'analyse étendue à l'ensemble des constructions et en tenant compte d'une stricte association pan de bois / tuile plate ne concerne que 25 bâtiments, soit environ $5 \%$ des constructions existantes. Au contraire, l'emploi de brique creuse, parpaing, tôle, fer et crépi est identifié dans plus de $20 \%$ des constructions. Ces matériaux moins «nobles» composent l'ossature des hangars agricoles et des constructions destinées à l'habitat principal sous la forme de pavillons et de leurs annexes. Le déclin des matériaux traditionnels au profit de matériaux moins chers, plus rapides et moins pénibles à poser ne constitue pas une surprise. Le constat apporte néanmoins la preuve d'un déséquilibre grandissant entre les constructions en matériaux modernes et les constructions en matériaux traditionnels.

Le second enseignement concerne la multiplication de l'association, sur les mêmes bâtiments, de matériaux d'époques différentes. Elle résulte de travaux de restauration d'habitations principales ou de résidences secondaires. Elle est plus fréquente encore dans la cas de la consolidation de bâtiments d'exploitation et des petits bâtiments annexes en pan de bois. Dans ce cas, le parpaing ou la brique creuse renforcent les murs en cours de désagrégation; les tôles métalliques ou en fibro-ciment se greffent à une toiture en tuile plate ou en ardoise trop longtemps restée sans entretien et que le vent a commencé à emporter. 


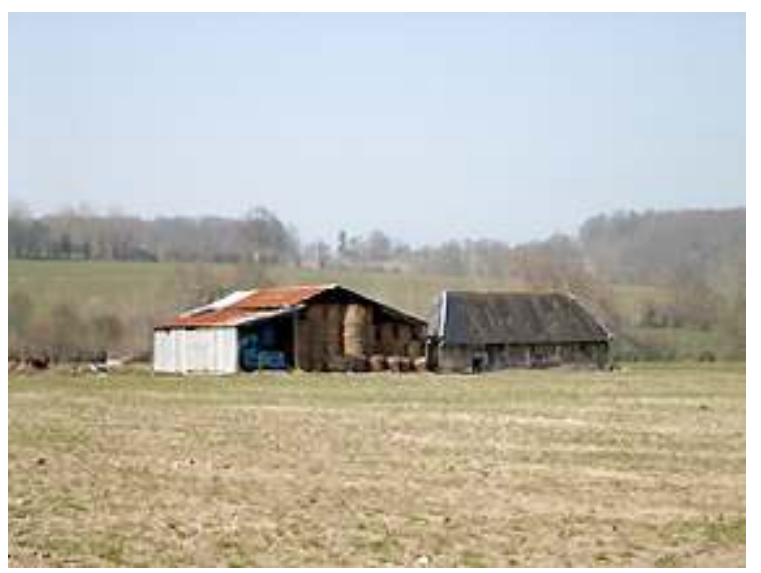

Bâtiments agricoles à Camembert : une juxtaposition de styles

Phot. M. Marie @ ( M. Marie, 2004

11 Le troisième élément significatif du repérage montre la juxtaposition de plus en plus fréquente des constructions de styles et d'époques différents (fig. $\mathbf{n}^{\circ} 3$ ). Ainsi, à proximité des restes du clos augeron, les constructions les plus récentes - pavillons et leurs annexes, bâtiments d'exploitation agricole - s'individualisent très fortement dans le paysage. Du fait de leur localisation, le plus souvent à proximité des voies de communication principales, et plus encore par leur emprise spatiale, ces constructions les plus récentes attirent le regard. La juxtaposition des styles s'exprime surtout dans l'enceinte même des exploitations agricoles. Désormais, les bâtiments en parpaing recouverts de tôles métalliques ou fibro-ciment, à la morphologie adaptée aux activités agricoles, côtoient dans le même ensemble les matériaux traditionnels de l'habitation principale et de quelques petites constructions reliques du clos augeron. Cet assemblage hétéroclite tourne toujours à l'avantage des premiers qui, par leur localisation, leur volume et leurs coloris, attirent l'œil.

Enfin, la multiplication des ruines constitue le dernier paramètre des transformations les plus marquantes. Au-delà des bâtiments considérés comme dégradés (environ $30 \%$ du total des constructions) dont plus des $2 / 3$ en pan de bois, de nombreuses ruines parsèment la commune. Certains éléments du clos augeron tels que la fromagerie ou la boulangerie en constituent l'essentiel. Inutilisés dans le cadre de l'activité agricole d'aujourd'hui, ces bâtiments sont une charge que de nombreux exploitants ne peuvent assumer. Une lente agonie ou, au contraire, une seconde vie à la faveur d'une vente pour démontage, sont le lot de ces petits édifices qui ne rentrent pas dans la rubrique des constructions dignes d'intérêt. Aux multiples ruines, il faut ajouter d'autres «nuisances visuelles» telles que les constructions non cadastrées (en bois et tôles rouillées), les carcasses de voitures abandonnées et les amas de déchets imputrescibles.

La dégradation architecturale et paysagère des campagnes méridionales du pays d'Auge procède donc de plusieurs échelles d'analyse : à l'échelle des bâtiments, des ensembles de constructions distribuées dans un même périmètre (sièges d'exploitation et hameaux) et aux échelles communale et intercommunale. 


\section{Une nouvelle géographie de l'habitat}

14

Deux évolutions antagonistes permettent d'expliquer une nouvelle géographie de l'habitat. La première est liée à une régression des constructions traditionnelles. Entre les relevés cadastraux de 1938 et l'enquête récente, on constate la disparition complète de 75 bâtiments, soit environ 14 \% des unités bâties repérées auxquelles il faut ajouter 10 ruines effondrées et 67 ruines debout. Au total, ce sont donc 152 bâtiments disparus ou appelés à disparaître soit près de $28 \%$ des constructions de la commune. La régression des constructions traditionnelles concerne principalement les bâtiments à usage agricole : conséquence directe de la diminution des exploitations et de l'inutilité ou de l'inadaptation des bâtiments pour l'agriculture actuelle. N'étant pas entretenus, ils se sont progressivement dégradés et nombre d'entre eux se sont effondrés. La disparition de ces bâtiments a aussi résulté de leur vente après démontage pour agrémenter le cadre paysager de résidences néorurales ou des résidences secondaires.

Parallèlement à la disparition de ces bâtiments traditionnels, apparaissent de nouvelles constructions qui répondent aux fonctions agricoles modernes et à l'habitat sous la forme de pavillons. Les premières accompagnent le plus souvent d'anciennes maisons d'habitation en partie débarrassées de leurs dépendances devenues inutilisables et «encombrantes». Quant aux secondes, elles sont érigées à la faveur d'une légère augmentation de la population communale liée à la proximité de Vimoutiers, le cheflieu de canton. La représentation cartographique de ces deux évolutions permet de mettre en évidence une nouvelle distribution des constructions. Il en résulte une «linéarisation » de l'habitat qui procède d'une part, de la raréfaction des constructions les plus isolées et d'autre part, de l'implantation des constructions récentes qui épouse les axes de communication les plus importants situés à proximité de Vimoutiers (fig. $\left.n^{\circ} 4\right)$. 
Figure 4

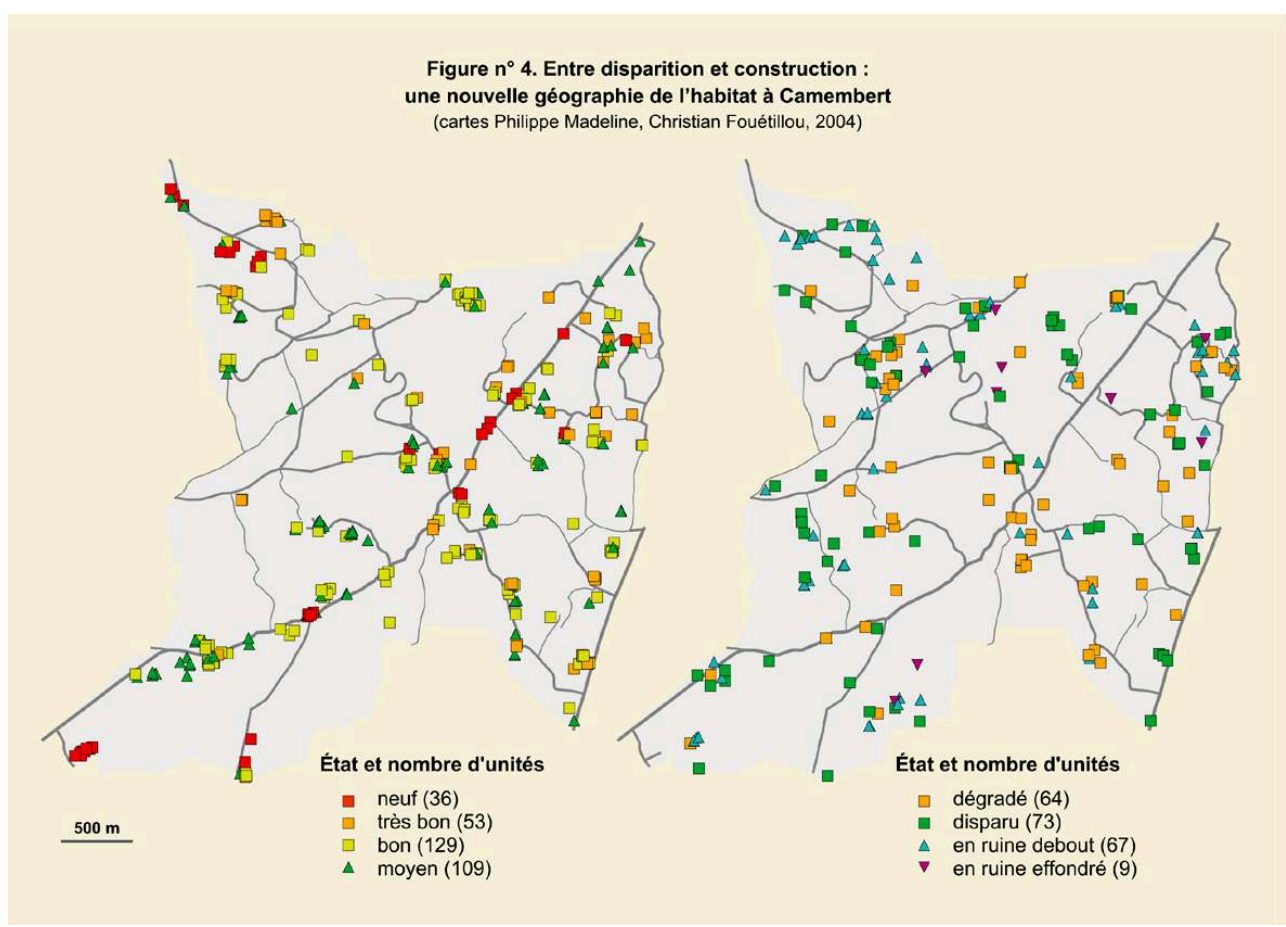

Entre disparition et construction : une nouvelle géographie de l'habitat à Camembert

Cartes P. Madeline - C. Fouétillou @ P. Madeline - C. Fouétillou, 2004

La diminution du nombre de bâtiments disséminés dans les herbages combinée au développement de nouvelles constructions à la localisation très sélective engendrent sinon une concentration de l'habitat, du moins la fin de sa très forte dispersion. La conjonction de ces deux tendances modifie les caractéristiques architecturale et paysagère de cet espace. Un constat renforcé par la législation sur la mise aux normes des bâtiments d'élevage.

\section{De nouveaux bâtiments d'élevage pour une nouvelle « monumentalité » rurale}

17 En quelques décennies, la modernisation de l'agriculture a modifié les paysages agraires et introduit l'idée d'un recyclage de plus en plus rapide de l'outil de travail dont les bâtiments d'élevage. Depuis 1993, la mise en application d'un programme de maitrise des pollutions d'origine agricole (PMPOA) censé réduire la dégradation de la qualité des eaux générée par les activités agricoles constitue un nouveau facteur de transformation des paysages. Avec l'obligation faite aux exploitants d'adapter leurs équipements et leurs pratiques en vue de la maîtrise des pollutions agricoles, des travaux pour la récupération et le stockage de la totalité des effluents d'élevage (fumier et lisier) s'imposent aux éleveurs. Selon les cas, les exploitants adaptent leurs bâtiments anciens ou en créent de nouveaux.

La réalisation du programme observé sur quatre cantons du nord-est du département de l'Orne (cantons d'Exmes, Gacé, Trun et Vimoutiers) montre que les aides publiques ont soutenu financièrement ce qui est obligatoire pour la mise aux normes, c'est-à-dire l'adaptation de l'outil de travail mais pas le coût des nouveaux bâtiments d'élevage, à la 
charge de l'exploitant. C'est ce qui explique leur rareté: sur les 76 dossiers subventionnés par les pouvoirs publics, à peine un tiers a donné lieu à des travaux de grande envergure correspondant au renouvellement complet de l'outil de travail. Les nouveaux bâtiments d'élevage répondent d'abord à un objectif environnemental mais participent aussi à une amélioration des conditions de travail des exploitants (mécanisation des tâches, gain de temps, amélioration des conditions d'hygiène sans négliger l'amélioration du bien-être animal) ainsi qu'à la recherche d'une meilleure productivité. La dimension sociale de cette nouvelle législation apparaît au grand jour et, à l'aune de ce que pouvait représenter autrefois le tas de fumier, les nouveaux bâtiments d'élevage constituent un marqueur social fort sans pour autant être un signe de vitalité des exploitations. Mais c'est la dimension paysagère de ces bâtiments d'élevage qui retient plus encore notre attention.

Figure 5



Un exemple de mise aux normes des bâtiments d'élevage : exploitation de Beaumoncel à Camembert Phot. P. Madeline (c) P. Madeline, 2002

Selon les choix réalisés par les exploitants, l'adaptation des bâtiments existants ou la création de bâtiments neufs et fonctionnels, les effets sur le paysage présentent des différences notables. Dans le premier cas, l'adaptation se résume souvent à la couverture des aires d'exercice. Il en résulte un enchevêtrement de toitures aux coloris et aux pentes différentes qui amplifie la juxtaposition déjà effective des formes, des matériaux et des couleurs. Dans le second cas, les nouveaux bâtiments créent une rupture avec les bâtiments des années 1960-1970 du fait de leurs formes, de leur volume et de leur couleur (fig. $\mathbf{n}^{\circ}$ 5). L'exemple d'une exploitation située dans le bourg de la commune de Camembert apporte un exemple assez éloquent de ce que l'on peut qualifier de nouvelle monumentalité rurale. Pour une exploitation de 85 vaches laitières, 30 génisses et 60 bovins à viande, l'agriculteur a fait construire une 
stabulation de $2537 \mathrm{~m}^{2}$ de superficie au sol et de 10 mètres de hauteur. Dans le paysage vallonné du pays d'Auge, le regard est inévitablement happé par ces nouvelles constructions. La singularité de ces nouveaux bâtiments ne réside pas seulement dans leurs volumes, elle s'exprime aussi dans leur aspect extérieur: la pente des toits qui contraste avec les bâtiments de type traditionnel; le choix des matériaux de construction, pour l'anecdote, qualifiés de " traditionnels » par l'architecte qui a visé le dossier. Même si les aspects esthétiques ne sont pas négligés, en témoignent un bardage en bois à claire-voie qui cache les rangées de parpaings dans leur partie supérieure, la toiture, en tôle teintée ou en panneaux de fibrociment naturel et tôle translucide qui présente l'avantage de mieux vieillir que la tôle galvanisée dont la brillance initiale puis la rouille attiraient le regard, enfin, les portes, en acier pré-laqué, qui apportent une touche colorée à l'ensemble de l'édifice.

Si, selon l'architecte Marc Verdier, les réalisations actuelles sont «moins pire que ce que l'on a pu connaître dans les années 1970 à $1990 »^{3}$ dans la mesure où l'on a commencé à se préoccuper de l'insertion paysagère des constructions, elles s'imposent désormais dans les communes rurales et concurrencent les édifices religieux dans les points de repères visuels du bocage augeron. Elles marquent, symboliquement, la suprématie de l'individualisme face à l'édifice qui incarnait la communauté villageoise.

Ainsi, ces nouveaux bâtiments d'élevage qui constituent une réponse à des enjeux agricoles (augmentation de la taille des exploitations, développement de la productivité et de la mécanisation des tâches) et à des préoccupations environnementales (préservation des ressources en eau et des paysages, bien-être animal) appellent les constats suivants : depuis la Seconde Guerre mondiale, on assiste à une accélération du rythme de construction des bâtiments agricoles et ces constructions reflètent de nouvelles préoccupations de la société.

\section{Une nouvelle « temporalité architecturale »}

Comme de nombreux exemples ont pu le montrer, la transformation de l'habitat rural n'est pas un phénomène lié uniquement à l'urbanisation et à la modernisation des campagnes qui a suivi la Seconde Guerre mondiale ${ }^{4}$. Sans entrer dans les détails, on identifie trois périodes majeures de construction pour le pays d'Auge entre le début du $\mathrm{XVI}^{\mathrm{e}}$ siècle et le milieu du XX $\mathrm{XX}^{\mathrm{e}}$ siècle. La première, après la guerre de Cent Ans, au début $\mathrm{du} \mathrm{XVI}^{\mathrm{e}}$ siècle, correspond à l'agrandissement des fermes seigneuriales et à la construction de bâtiments spécialisés: écuries, étables, bergeries, granges, charreteries. La deuxième correspond au couchage en herbe du pays d'Auge, à partir du milieu du XVIII ${ }^{e}$ siècle: c'est une période de réorganisation des bâtiments d'exploitation avec l'abandon des granges ou leur reconversion, la construction d'étables ou leur agrandissement, l'édification des bouveries et des pressoirs. Cette période de création des fermes manoirs, des maisons modestes et de leurs petites annexes est suivie par l'entrée de l'agriculture augeronne dans la voie de l'intensification agricole, notamment à partir de 1966, date de mise en application par les pouvoirs publics de soutiens financiers à la construction d'outils de travail modernes. Enfin, avec l'application du PMPOA en 1993 puis la seconde phase du programme à partir de 2003, une nouvelle génération de bâtiments d'élevage voit le jour. L'exemple de la ferme de Beaumoncel située à Camembert est une bonne illustration de ce changement dans le rythme des constructions (fig. $\mathbf{n}^{\circ} \mathbf{6}$ ). 




Un exemple d'accélération du rythme des constructions agricoles : l'exploitation de Beaumoncel à Camembert

Cartes P. Madeline - C. Fouétillou (C P. Madeline - C. Fouétillou, 2004

La deuxième moitié du $\mathrm{XX}^{\mathrm{e}}$ siècle constitue donc un changement dans le rythme des constructions avec une accélération de ce rythme qui répond soit à des incitations soit à des réglementations. On peut même parler de rupture car les réalisations n'obéissent pas seulement à l'arrivée de nouvelles techniques de construction ou de nouveaux matériaux mais relèvent de considérations extérieures à la sphère agricole. C'est l'expression de nouvelles préoccupations de la société face aux débordements de quatre décennies de productivisme agricole qui obligent désormais les agriculteurs à intégrer des aspects non productifs.

En effet, la législation sur les paysages de 1992 et 1994 impose désormais, dans le dossier de permis de construire, la rédaction des documents relatifs à la prise en compte du paysage. Les documents joints au permis de construire (notice d'impact visuel du projet, documents graphiques et photographiques avec coupe de terrain) doivent expliquer l'impact du projet à court, moyen et long termes. De plus, pour les installations classées, une analyse de risque "d'atteinte au paysage» implique des mesures compensatoires qui se résument à des plantations pour masquer la construction, le choix des matériaux, l'implantation du bâtiment. Il s'agit au total, selon les termes des "techniciens bâtiment " des chambres d'agriculture «de veiller à une bonne insertion paysagère des nouveaux bâtiments ». Des actions supplémentaires, absentes pour la première phase du PMPOA du département de l'Orne, permettent aux exploitants d'améliorer l'esthétique de leurs bâtiments et leurs abords ${ }^{5}$. Ainsi, les changements par rapport à la vague de construction précédente sont nets: ils 
concernent l'introduction de matériaux « plus doux » et « plus chauds » tels que le bois et la brique à la place des murs en aggloméré non enduits; l'utilisation (rare) de produits imitant la tuile ou plus généralement la coloration des tôles fibrociment à la place du gris et de la tôle nue étincelante; la disposition horizontale ou verticale du bardage afin de jouer sur la perception de la hauteur et de la longueur du bâtiment mais aussi les efforts de replantation, de décoration et de propreté6.

Ces dispositions censées donner satisfaction aux attentes de la population ne peuvent être ignorées mais posent un problème plus général de réflexion sur l'architecture des bâtiments d'élevage.

\section{Conclusion}

Les impacts des transformations agricoles et rurales de la deuxième moitié du $\mathrm{XX}^{\mathrm{e}}$ siècle sur le bâti du pays d'Auge méridional sont multiples :

- une inversion progressive du rapport entre les bâtiments en matériaux traditionnels et les constructions récentes, qu'il s'agisse de bâtiments agricoles ou d'habitations principales sous la forme de pavillons ;

- l'importance visuelle des nouvelles constructions (pavillons ou hangars agricoles) qui sont installées en priorité le long des axes de communication les plus fréquentés et sont donc, par conséquent, les plus facilement repérées ;

- une modification de la répartition de l'habitat résultant de mouvements antagonistes: création de nouvelles constructions (pavillons, bâtiments d'élevage aux normes environnementales) et disparition de bâtiments agricoles inutiles pour l'agriculture d'aujourd'hui ;

- le caractère hétéroclite des matériaux utilisés, généralement de couleurs vives, plus aisément repérables que les bâtiments de type traditionnel. L'individualisation des constructions dans le paysage est particulièrement forte dans le cas des bâtiments agricoles les plus récents dont le volume écrase littéralement le champ de vision;

- l'ouverture progressive des paysages par la réduction des haies et des plants de pommiers qui disparaissent au gré des agrandissements de parcelles et de l'extension de la superficie consacrée au maïs fourrager. Cette ouverture, à travers laquelle les constructions imposantes et voyantes accompagnées de leurs haies brise-vent constituées d'essences végétales étrangères à la région et au feuillage persistant, fixent le regard, est de plus en plus large et de plus en plus lointaine ;

- la multiplication de «nuisances visuelles» et la présence de nombreuses «tâches» particulièrement visibles lorsque la végétation est en dormance.

Ce constat n'est assurément pas propre au pays d'Auge méridional. Mais dans cet espace si bien identifié et médiatisé au point de fournir une image idyllique et rassurante d'un territoire que l'action des hommes aurait miraculeusement épargné, le décalage avec la réalité est flagrant. Peut-on évoquer un éventuel préjudice par rapport aux possibilités de développement de nouvelles activités rurales?

Malgré les exemples de valorisation du patrimoine comme support d'un tourisme culturel $^{7}$, il est difficile de répondre à la question. Toutefois, l'analyse souligne le paradoxe suivant: malgré la diminution du nombre des exploitations agricoles, les bâtiments qui les accompagnent sont de plus en plus visibles dans le paysage, qu'il s'agisse de la construction de "monuments agricoles" reflétant socialement les champions de la compétition agricole engagée ou de l'assemblage hétéroclite de 
bâtiments de générations successives mêlant des formes, des matériaux et des couleurs différentes. Dans des espaces escarpés comme ceux du pays d'Auge où la disparition des haies ouvre des perspectives paysagères sans cesse élargies, l'absence de réflexion dans la construction des bâtiments d'élevage est criante. Marc Verdier, un des rares architectes intéressé par les bâtiments d'élevage, résume bien la situation: "Finalement, on remplace la banalité non recevable (les préfabriqués en mauvaise tôle et les couleurs de récupération ...) par de la banalité recevable, consensuelle et négociée ${ }^{8} » . A u$ regard des constructions passées dont la plus-value patrimoniale constitue un vecteur des politiques de développement local, il ne s'agit pas de préconiser la construction de bâtiments (pavillons, bâtiment d'élevage, locaux d'entreprises) selon les plans, les formes et les matériaux d'hier mais de réfléchir à des créations paysagères qui pourraient mieux s'inscrire dans leur temps et dans leur espace.

Pourquoi les bâtiments agricoles ne méritent-ils pas plus d'attention de la part des architectes et des aménageurs au-delà d'un simple habillage végétal qui doit « absorber » les constructions modernes dans leur environnement? La construction agricole qui représente aujourd'hui $35 \%$ des surfaces bâties non résidentielles est encore trop ignorée des architectes. La primauté des aspects fonctionnels conduit ainsi irrémédiablement à une banalisation des bâtiments d'élevage sur la totalité du territoire. Sans tomber dans une position excessive qui conduirait à figer des paysages, des efforts de réflexion et de création de bâtiments d'élevage ne devraient plus être des exceptions médiatisées par les brochures des ministères de l'Agriculture et de la Culture mais être généralisées ${ }^{9}$. Dans une région agricole où les conditions naturelles ont freiné la mise en place d'une agriculture productiviste - en témoigne le classement en «zones agricoles défavorisées et de montagne »-, des perspectives agricoles et surtout rurales plus respectueuses des paysages ne sont-elles pas des pistes de développement à explorer ${ }^{10}$ ?

\section{BIBLIOGRAPHIE}

Bonnoin, Philippe, Perrot, M., de la Soudiere, M. L'ostal en Margeride. CNRS, 1983.

Brunet, Pierre, Girardin, Pierre. Inventaire Régional des Paysages de Basse-Normandie. Conseil Régional de Basse-Normandie, DIREN. cédérom, 2002.

Chambre d'Agriculture du Calvados et le CAUE. cédérom intitulé Bâtiments agricoles et paysage, novembre 2000 .

Madeline, Philippe. L'état du patrimoine bâti à Camembert. Un révélateur de transformations paysagères au sud du pays d'Auge. Dans Autour de Camembert de l'An Mil à l'an 2000. Quatre années de recherches interdisciplinaires. Enquêtes Rurales, 6, Cahiers de la MRSH de Caen, XIX, PUC, Université de Caen Basse-Normandie, 1999, p. 173-186.

Madeline, Philippe. Le renouvellement des bâtiments d'élevage dans le sud pays d'Auge : un indice des transformations agricoles et rurales. Les Paysages ruraux en Normandie. Actes du 
$37^{\mathrm{e}}$ Congrès de la Fédération des Sociétés Historiques et Archéologiques de Normandie

(Pont-Audemer, octobre 2002). Caen : Annales de Normandie, 2003, p. 423-434.

Ministère de l'Agriculture, de la Pêche et des Affaires rurales et ministère de la Culture et de la Communication. La Qualité architecturale des bâtiments d'élevage. Février 2003.

Mousset, Hélène, Lecherbonnier, Yannick. Inventaire général des monuments et des richesses artistiques de la France. Canton de Vimoutiers. Orne. Service régional de l'Inventaire de BasseNormandie, 1994 (Collection Images du Patrimoine, $\mathrm{n}^{\circ}$ 132).

Verdier, Marc. Bâtiments agricoles : le besoin d'architecture. Aménagement et Nature, $\mathrm{n}^{\circ} 141$, juin 2001, p. 107-116.

\section{NOTES}

1. Pôle rural : sociétés, paysages, environnement. Maison de la Recherche en Sciences Humaines de l'Université de Caen Basse-Normandie. Esplanade de la paix. 14032 Caen Cedex. http:// www.unicaen.fr/mrsh/index.php

2. Mousset, Hélène, Lecherbonnier, Yannick. Canton de Vimoutiers. Orne. Direction régionale des affaires culturelles de Basse-Normandie, service régional de l'Inventaire, 1994 (Coll. Images $\mathrm{du}$ Patrimoine, $\mathrm{n}^{\circ}$ 132).

3. Verdier, Marc. Bâtiments agricoles : le besoin d'architecture. Aménagement et Nature, $n^{\circ} 141$, juin 2001, p. 109.

4. Bonnoin, Philippe, Perrot, M., de la Soudiere, M. L'ostal en Margeride. CNRS, 1983.

5. Le Conseil Général du Calvados apporte un concours financier à la coloration des toitures des bâtiments agricoles.

6. On peut notamment se référer à une étude réalisée par la Chambre d'Agriculture du Calvados et le CAUE. Cédérom intitulé Bâtiments agricoles et paysage, novembre 2000.

7. Colloque organisé en 1997 par l'Institut d'Etudes Supérieures des Arts (IESA) et la DATAR. Paris.

8. Verdier, Marc. Bâtiments agricoles : le besoin d'architecture. Aménagement et Nature, $n^{\circ} 141$, juin 2001, p. 109.

9. «La qualité architecturale des bâtiments d'élevage », brochure du ministère de l'Agriculture, de la Pêche et des Affaires Rurales et du ministère de la Culture et de la Communication, février 2003.

10. Cette problématique fut au centre des débats du colloque «Protection, restauration et aménagement du patrimoine agricole bâti et Europe ", qui se tint à La Garenne-Lemot (LoireAtlantique) en mai 2005.

\section{RÉSUMÉS}

Partant de l'exemple de la commune de Camembert dans le pays d'Auge, zone bénéficiant de l'image archétypique d'une campagne préservée, cet article se propose d'évaluer l'impact sur le paysage des modifications du bâti induites par l'évolution de la population et les transformations de l'économie agricole. L'abandon ou la transformation du bâti ancien, la multiplication des 
constructions neuves à usage d'habitation ou destinées à l'élevage constituent d'importants facteurs de modification du paysage et nous invitent à nous interroger sur leur devenir.

Starting out from the commune of Camembert in the pays d'Auge in Normandy, a place which enjoys an archetypal image of a well-preserved piece of countryside, this article sets out to evaluate the effects on the landscape of the modifications in built forms resulting from changes in population and transformations in the agricultural economy. The abandonment or transformation of old buildings and the multiplication of new constructions either for housing or for livestock rearing are important factors in the way the landscape is changing and constitutes an invitation to reflect on the future of this landscape.

\section{INDEX}

Keywords : Camembert, pays d'Auge, Normandy, landscape, building materials, geography, rural dwellings, livestock buildings, agricultural constructions, farm buildings, rural architecture Mots-clés : Camembert, pays d'Auge, Normandie, paysage, matériaux de construction, géographie, habitat rural, bâtiment d'élevage, construction agricole, bâtiment agricole, architecture rurale

\section{AUTEUR}

\section{PHILIPPE MADELINE}

Maître de conférences en géographie, Université de Caen Basse-Normandie. Co-directeur du Pôle Rural de la Maison de la Recherche en Sciences Humaines/Umr ESO 6590 - CRESO Caen.

madeline@mrsh.unicaen.fr 